Behav. Sci. 2014, 4, 70-71; doi:10.3390/bs4010070

Editorial

\title{
Acknowledgement to Reviewers of Behavioral Sciences in 2013
}

\section{Behavioral Sciences Editorial Office}

MDPI AG, Klybeckstrasse 64, CH-4057 Basel, Switzerland

Published: 26 February 2014

The editors of Behavioral Sciences would like to express their sincere gratitude to the following reviewers for assessing manuscripts in 2013:

$\begin{array}{lll}\text { Alho, Päivi } & \text { Deshmukh, Vinod } & \text { Jimeno, Natalia } \\ \text { Allen, Andrew } & \text { Devonis, David C. } & \text { Kak, Subhash } \\ \text { Aretouli, Eleni } & \text { Diestel, Stefan } & \text { Kamens, Helen } \\ \text { Beebe, John } & \text { Eddington, Kari M. } & \text { Keller, Andreas } \\ \text { Blasche, G. } & \text { Elliot, Andrew } & \text { Kitamura, Christine } \\ \text { Burnham, John C. } & \text { Elmore, Lauren Caitlin } & \text { Krishnan, Mohan } \\ \text { Butler, David L. } & \text { Fidalgo, C. } & \text { Lane, Andrew } \\ \text { C'de Baca, Janet } & \text { Fleming, Jennifer } & \text { Lany, Jill } \\ \text { Caillouet, Beth } & \text { Frasca, Diana } & \text { Li, Xia } \\ \text { Cambray, Joseph } & \text { Frowd, Charlie D. } & \text { Lienard, Pierre } \\ \text { Cardo, Esther } & \text { Gawęda, Lukasz } & \text { Maack, Danielle } \\ \text { Carrera, Pilar } & \text { Gilbert, Avery } & \text { Macdonald, Kevin } \\ \text { Chang, Luke J. } & \text { Guo, Gao } & \text { Mackay, Harry A. } \\ \text { Chee, Christine } & \text { Harasymchuk, Cheryl } & \text { Maclean, Evan L. } \\ \text { Clair, Michelle C. St } & \text { Hartley, James } & \text { Main, Roderick } \\ \text { Clapper, John } & \text { Hashimoto, Ryu-Ichiro } & \text { Martyr, Anthony } \\ \text { Clay, Andrea W. } & \text { Hinton, W. Ladson } & \text { Matthews, Gerald } \\ \text { Craddock, Travis } & \text { Hirvonen, Riikka } & \text { Mcgann, Marek } \\ \text { Danckert, James } & \text { Hodgson, Tim } & \text { Mills, Jon } \\ \text { Darlington, Beth } & \text { Hoff, Robert } & \text { Morimura, Naruki } \\ \text { Dawson, Terence } & \text { Ishitobi, Makoto } & \text { Morioka, Masayoshi } \\ \text { De la Torre, Gabriel G. } & \text { Jasper, Fabian } & \text { Muse, Mark }\end{array}$




$\begin{array}{lll}\text { Näätänen, Risto } & \text { Roesler, Christian } & \text { Susswein, Noah } \\ \text { Nadler, Ruby T. } & \text { Rosegrant, John } & \text { Szpunar, Karl } \\ \text { Niznikiewicz, Margaret } & \text { Saban, Mark } & \text { Talpos, John } \\ \text { Nolan, Steve } & \text { Saksida, Lisa M. } & \text { Topolovec-Vranic, Jane } \\ \text { Nowicki, Steve } & \text { Schipul, Sarah E. } & \text { Turner, Robert } \\ \text { Oblak, Adrian L. } & \text { Schmitter-Edgecombe, Maureen } & \text { Udal, Anne H } \\ \text { Oertel, Viola } & \text { Schott, Björn H. } & \text { Uher, Jana } \\ \text { Oostenbroek, Janine } & \text { Segal, Robert } & \text { Van Der Lugt, Arie } \\ \text { Pauly, Katharina } & \text { Shakeel, Mohammed Kalathil } & \text { Van Tilburg, Wijnand A. P. } \\ \text { Peet, Christopher } & \text { Sharp, Carla } & \text { Walach, Harald } \\ \text { Petrazzini, Maria Elena Miletto } & \text { Sheehy, Maura } & \text { Walkerdine, Valerie } \\ \text { Ploeger, Annemie } & \text { Smythe, William } & \text { Warburton, E.C. } \\ \text { Price, Katy } & \text { Snowden, Charles } & \text { Weishaus, Joel } \\ \text { Ragland, Daniel } & \text { Sparrow, G. Scott } & \text { Williams, Lisa } \\ \text { Ravizza, Susan M. } & \text { Stein, Murray } & \text { Zoladz, Phillip R. } \\ \text { Reardon, Annemarie } & \text { Stone, Ws } & \\ \text { Reidy, Dennis E. } & \text { Storm, Lance } & \end{array}$

(C) 2014 by the authors; licensee MDPI, Basel, Switzerland. This article is an open access article distributed under the terms and conditions of the Creative Commons Attribution license (http://creativecommons.org/licenses/by/3.0/). 